\title{
Prognostic value of Notch-I expression in hepatocellular carcinoma: a meta-analysis
}

This article was published in the following Dove Press journal:

OncoTargets and Therapy

22 October 2015

Number of times this article has been viewed

Tao $\mathrm{Wu}^{\prime}$

Min Jiao'

Li Jing'

Min-Cong Wang'

Hai-Feng Sun ${ }^{2}$

Qing $\mathrm{Li}^{\prime}$

Yi-Yang Bai'

Yong-Chang Wei'

Ke-Jun Nan'

Hui Guo'

'Department of Medical Oncology, The First Affiliated Hospital of Xi'an Jiaotong University, ${ }^{2}$ Department of Oncology, Shaanxi Cancer Hospital, Xi'an, People's Republic of China

Correspondence: Ke-Jun Nan Department of Medical Oncology, The First Affiliated Hospital of Xi'an Jiaotong University, 277 Yanta West Road, Xi'an 7I006I, Shaanxi, People's Republic of China

Tel/fax +86 2985324086

Email nankj@।63.com

Hui Guo

Department of Medical Oncology, The First Affiliated Hospital of Xi'an jiaotong University, 277 Yanta West Road, Xi'an 71006I, Shaanxi, People's Republic of China

Email guohuihappy97@I63.com
Abstract: Association of Notch-1 expression with prognosis of patients with hepatocellular carcinoma (HCC) remains controversial. We conducted a meta-analysis to reevaluate the association of Notch-1 expression with clinicopathological characteristics and prognosis of HCC. PubMed, Embase, Web of Science, and China National Knowledge Infrastructure were searched to look for relevant studies. The association between Notch-1 expression and clinicopathological parameters and overall survival (OS) was then reassessed using the meta-analysis for odds ratio (OR) or hazard ratio (HR) and 95\% confidence interval (CI). A total of seven studies, including $810 \mathrm{HCC}$ patients, were eligible for the meta-analysis. Our data showed that high Notch-1 expression was able to predict poor OS (HR 1.50, 95\% CI 1.17-1.83, $P=0.0001$ ). The pooled OR showed that high Notch-1 expression was significantly associated with tumor metastasis (OR $0.37,95 \% \mathrm{CI} 0.16-0.86, P=0.02)$ and tumor size $>5 \mathrm{~cm}(\mathrm{OR} 0.48,95 \% \mathrm{CI}$ $0.26-0.88, P=0.02)$. In contrast, there was no association between high Notch-1 expression and tumor differentiation, late TNM stage, tumor number, and portal vein invasion of HCC. In conclusion, Notch-1 overexpression might predict poorer survival and more aggressive behavior in patients with HCC.

Keywords: hepatocellular carcinoma, Notch-1, prognosis, clinicopathological features, meta-analysis

\section{Introduction}

Liver cancer is a lethal and aggressive neoplasm representing the fifth most commonly diagnosed malignancy in men and the ninth in women globally; it is also a leading cause of cancer-related death. ${ }^{1,2}$ Hepatocellular carcinoma (HCC) and intrahepatic cholangiocarcinoma account for the majority of primary liver malignancies, in which HCC represents the major histological subtype ( $>80 \%$ of primary liver cancers). ${ }^{3}$ $\mathrm{HCC}$ is well characterized as a highly refractory malignancy associated with rapid tumor progression and metastasis, resulting in a 5-year survival rate as low as $16 \%{ }^{4}$ Therefore, efforts to identify new additional prognostic and predictive markers should be made to improve individual treatment strategies and prognosis in HCC.

Accumulated evidence shows that the Notch signaling pathways play a significant role in HCC progression, and could be used as therapeutic targets for the patients. A variety of potential therapies exist to modulate Notch signaling in HCC, including neutralizing antibodies, siRNAs, and miRNA therapies, which target specific Notch tumorigenic subunits..$^{5}$ Indeed, the Notch signaling is a highly evolutionally conserved pathway that regulates cell fate, proliferation, apoptosis, differentiation, and survival, and dysregulation of this pathway is the basis of different diseases, including cancer. ${ }^{6}$ However, mounting evidence indicates that the Notch signaling pathway can exert oncogenic or tumor-suppressive action in various cancers. ${ }^{7}$ For example, 
the oncogenic potential of the Notch activation is implicated in breast, colorectal, and melanoma cancers. On the other hand, it can also be tumor suppressive in HCC, as well as in head and neck squamous cell carcinoma. ${ }^{8}$ Furthermore, the Notch pathway consists of four transmembrane receptors (Notch-1, -2, -3, and -4) and five ligands that include the Jagged family (Jagged1 and Jagged2) and Delta-like family (DLL1, 3, and 4) in mammals. ${ }^{9}$ When these ligands bind to Notch receptors, a $\gamma$-secretase complex then mediates the transmembrane domain cleavage of the Notch receptor and the Notch intracellular domain (NICD). The released NICD then translocates into the nucleus and functions as a transcriptional coactivator to drive the expression of target genes. ${ }^{6}$ As an important receptor of Notch family, Notch-1 was first discovered through its involvement in T-cell acute lymphoblastic leukemia and T-cell leukemogenesis. To date, numerous studies have shown that high Notch-1 expression is associated with progression and prognosis of various tumors, including breast cancer, ${ }^{10,11}$ non-small-cell lung cancer, ${ }^{12,13}$ esophageal cancer, ${ }^{14}$ colorectal cancer, ${ }^{15,16}$ and ovarian cancer. ${ }^{17}$

Although Notch-1 is implicated in clinicopathological characteristics and prognosis of HCC, other studies ${ }^{18,19}$ did report conflicting results. Therefore, it remains unknown whether this discrepancy is caused by limited sample sizes or genuine heterogeneity. In this study, a meta-analysis was performed to assess the relationship between Notch-1 expression and clinicopathological parameters and clinical outcomes of HCC.

\section{Materials and methods}

\section{Literature search strategy}

This meta-analysis was reported according to the Preferred Reporting Items for Systematic Reviews and Meta-Analyses (PRISMA) statement. ${ }^{20}$ The following electronic databases were systematically searched: PubMed, Embase, Web of Science, and China National Knowledge Infrastructure. The search was conducted on June 1, 2015 and was limited to papers published in English and Chinese. Studies were selected using the combinations of the following search terms: (Notch OR Notch-1 OR Notch 1) AND (hepatocellular carcinoma OR hepatoma OR HCC OR liver cancer) AND (prognosis OR prognostic OR outcome OR mortality OR survival). To obtain some unavailable data from the eligible articles, we contacted some of the authors. The bibliographies of articles were also manually examined to identify additional studies. Two authors conducted the search and assessed the eligibility of studies independently. Any disagreements were resolved by iteration, discussion, and consensus between the two reviewers.

\section{Study selection}

The inclusion criteria were as follows: 1) cancer patients who were pathologically confirmed; 2) Notch-1 expression was evaluated in HCC tissues; 3) Notch-1 expression was examined by immunohistochemistry (IHC) or polymerase chain reaction (PCR); 4) studies analyzed the association of Notch-1 expression with HCC clinicopathological parameters or prognosis; 5) studies of prognosis association provided sufficient information to estimate hazard ratio (HR) for overall survival (OS) or disease-free survival (DFS) and $95 \%$ confidence interval (CI);6) sample size was more than 20 cases; 7) if there were multiple articles overlapping the same cohorts, only the most complete article was included. The exclusion criteria were as follows: 1) publication was of non-research articles; 2) studies had duplicate data or lack of key information for further analysis; 3) studies were based on animal or human cell lines.

\section{Data extraction}

All eligible publications were reviewed, and then data were extracted by two independent authors (Min-Cong Wang and Qing Li). The extracted data were then summarized in a consistent manner to prevent bias. The following information was extracted: name of the first author, year of publication, study population characteristics (patient number, age, sex), clinicopathological parameters (tumor size, metastasis, vein invasion, stage, differentiation), follow-up data (OS and DFS, Notch-1 assessment method, and cutoff value of Notch-1. HR was first extracted and synthesized from multivariable analysis where available. If such information was missing, we estimated the HR from Kaplan-Meier curves using the methods reported by Tierney et al. ${ }^{21}$

\section{Qualitative assessment}

Li Jing and Qing Li independently assessed the quality of each of the available studies using the Newcastle-Ottawa Quality Assessment Scale (NOS) with our reasonable modifications (Table S1). ${ }^{22}$ This scale uses a star system (a score of $0-9$ ) to indicate the quality of each study (Table 1). Studies labeled with six or more stars were considered to be of high quality.

\section{Statistical analysis}

All statistical analyses were carried out by Stata 12.0 (Stata Corporation, College Station, TX, USA) and Review 


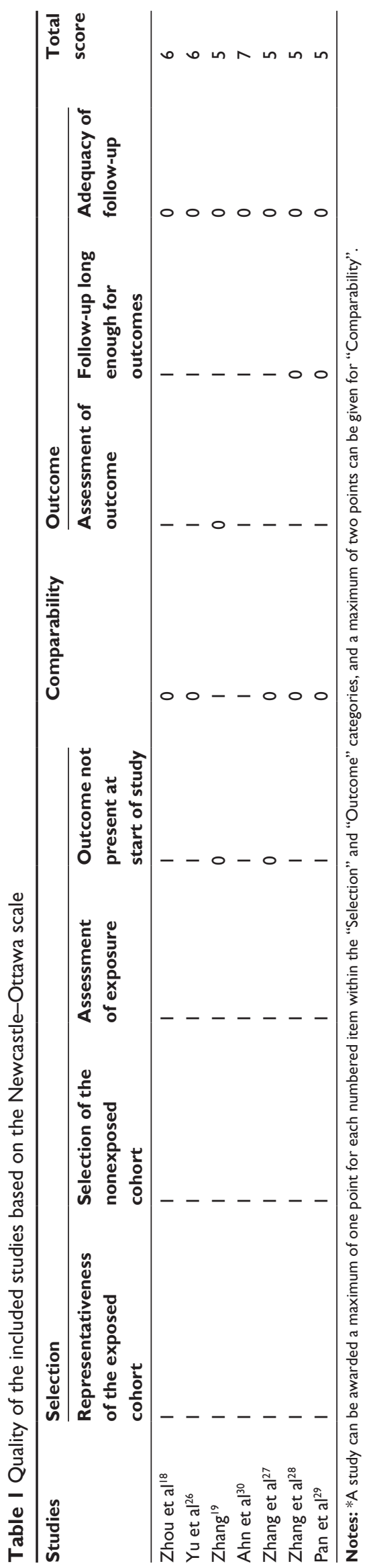

Manager 5.2 (Cochrane Collaboration, London, UK). Data on prognostic ability of Notch-1 expression predicting OS were pooled across studies. When these data were not directly provided in the eligible articles, we calculated the HR and its 95\% CI from Kaplan-Meier survival curve using Engauge Digitizer version 4.1 (free software downloaded from http://sourceforge.net). The estimated odds ratio (OR) was used to summarize the relationship between Notch-1 expression and the clinicopathological features of patients. In the present study, a combined HR $>1$ implied a worse prognosis in the group with high Notch-1 expression, while an $\mathrm{OR}<1$ indicated a higher probability of tumor progression in HCC with Notch-1 overexpression. There was no overlap of the $95 \%$ CI with 1 , with 1 indicating a statistical significance. In the course of data pooling, statistical heterogeneity was performed by using chi-square-based $Q$-test. The $I^{2}$ value indicated the degree of heterogeneity. A $P$-value $<0.10$ and/or $I^{2}>50 \%$ was considered significant heterogeneity, and a random-effects model (REM) was used. Otherwise, a fixed-effects model was used. ${ }^{23}$ Publication bias was assessed by Egger's and Begg's funnel plot test. ${ }^{24,25}$

\section{Results Characteristics of studied populations}

As shown in Figure 1, 125 records of the association of Notch-1 with HCC were identified via database searching. However, 108 studies were excluded because of duplicate reports, studies irrelevant to our aim, or studies without clinical specimens. Of the remaining 17 studies identified for further evaluation, ten were then excluded after full-text assessment due to insufficient data and duplicated cohort of patients. Eventually, seven studies were identified for the final meta-analysis. ${ }^{18,19,26-30}$ The major characteristics of the seven studies are summarized in Table 2. Specifically, they included a total of 810 patients from People's Republic of China and Korea with 585 males and 225 females. IHC and real-time PCR were used to detect Notch-1 expression in HCC specimens. The patients' age ranged from 48.5 to 66.7 years. Six studies defined the cutoff value of Notch-1 expression by combining the intensity and percentage, whereas only one study used the staining percentage of Notch-1 expression. DFS was estimated in one study, and OS was presented in four studies. All seven eligible studies evaluated the association of Notch-1 expression with tumor pathological features. HR and $95 \% \mathrm{CI}$ were directly obtained from four studies, and for the remaining study, they were extrapolated from Kaplan-Meier curves. According to NOS 


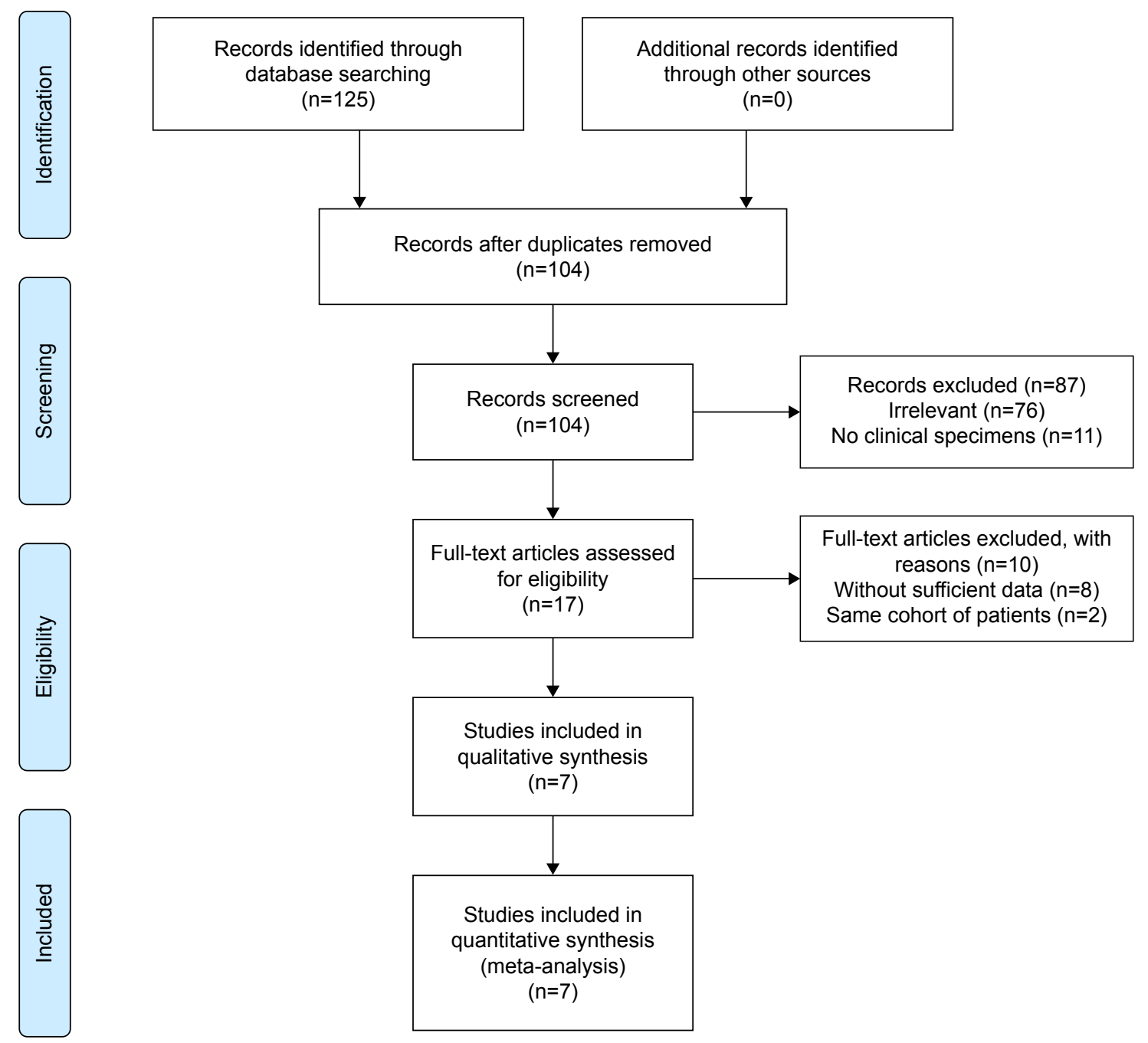

Figure I Flow diagram showing study selection procedure.

quality assessment, the scores of included studies ranged from 5 to 7 .

\section{Notch-I expression and OS of HCC patients}

Four of the seven studies had estimated association of Notch-1 expression with OS. As shown in Figure 2, pooled data from all these studies suggested that high Notch-1 expression was significantly associated with poor OS. The pooled HR was 1.50 (95\% CI 1.17-1.83, $P=0.0001$ ), indicating that higher Notch-1 expression predicted worse prognosis in HCC. No significant heterogeneity was observed ( $P=0.0 \%, P=0.579)$, so the fixed-effects model was used for further data analysis.

\section{Correlation of Notch-I expression and clinicopathological features}

Six studies reported the association of TNM stage with Notch-1 expression. The data were significantly heterogeneous $\left(P<0.00001, I^{2}=88 \%\right)$. Thus, an REM was used. The pooled OR revealed that high Notch-1 expression was not associated with tumor stages (OR $0.31,95 \%$ CI $0.08-1.15, P=0.08$; Figure $3 \mathrm{~A}$ ). Three studies investigated the relationship between Notch-1 expression and tumor number. ${ }^{18,26,27}$ Their pooled analysis showed that high Notch-1 expression was not connected to tumor number (OR 0.75, 95\% CI 0.48-1.16, $P=0.19$; Figure $3 \mathrm{~B}$ ), and no heterogeneity was found among these studies $\left(P=0.47, I^{2}=0 \%\right)$. All studies described Notch-1 expression according to tumor size. Heterogeneity was observed among these studies $(P=0.001$, $\left.I^{2}=72 \%\right)$. The results showed that Notch-1 overexpression was associated with larger tumor (size $>5 \mathrm{~cm}$ : OR $0.48,95 \%$ CI 0.26-0.88, $P=0.02$ using REM; Figure 3C).

Four studies investigated association of Notch-1 expression with HCC metastasis ${ }^{18,19,26,28}$ and showed that high Notch-1 expression was significantly associated with tumor metastasis, with a pooled OR estimate of 0.37 (95\% CI 
Table 2 Baseline characteristics of the studies in the meta-analysis

\begin{tabular}{|c|c|c|c|c|c|c|c|}
\hline Studies & Year & Country & Tumor type & $\begin{array}{l}\text { Sample size } \\
\text { (male/female) }\end{array}$ & $\begin{array}{l}\text { Mean age } \\
\text { (years) }\end{array}$ & $\begin{array}{l}\text { Vein invasion } \\
\text { (yes/no) }\end{array}$ & $\begin{array}{l}\text { Stage } \\
\text { (I-II/III-IV) }\end{array}$ \\
\hline Zhou et al ${ }^{18}$ & 2012 & $\begin{array}{l}\text { People's Republic } \\
\text { of China }\end{array}$ & $\mathrm{HCC}$ & $74 / 46$ & 48.5 & $26 / 94$ & $32 / 88$ \\
\hline Yu et $\mathrm{a}^{26}$ & 2014 & $\begin{array}{l}\text { People's Republic } \\
\text { of China }\end{array}$ & $\mathrm{HCC}$ & $70 / 62$ & 50.3 & $70 / 62$ & $49 / 83$ \\
\hline Zhang ${ }^{19}$ & 2010 & $\begin{array}{l}\text { People's Republic } \\
\text { of China }\end{array}$ & $\mathrm{HCC}$ & $33 / 7$ & NA & NA & $31 / 9$ \\
\hline Ahn et $\mathrm{al}^{30}$ & 2013 & Korea & $\mathrm{HCC}$ & $237 / 51$ & 52.6 & $13 / 275$ & $225 / 63$ \\
\hline Zhang et al ${ }^{27}$ & 2013 & $\begin{array}{l}\text { People's Republic } \\
\text { of China }\end{array}$ & $\mathrm{HCC}$ & $74 / 36$ & 66.7 & $26 / 84$ & $60 / 50$ \\
\hline Zhang et al ${ }^{28}$ & 2012 & $\begin{array}{l}\text { People's Republic } \\
\text { of China }\end{array}$ & $\mathrm{HCC}$ & $65 / 13$ & NA & NA & $35 / 43$ \\
\hline Pan et $\mathrm{al}^{29}$ & 2014 & $\begin{array}{l}\text { People's Republic } \\
\text { of China }\end{array}$ & $\mathrm{HCC}$ & $32 / 10$ & NA & NA & $38 / 4$ \\
\hline Studies & $\begin{array}{l}\text { Multivariate } \\
\text { analysis }\end{array}$ & $\begin{array}{l}\text { Tumor size } \\
(<5 \mathrm{~cm} />5 \mathrm{~cm})\end{array}$ & $\begin{array}{l}\text { Analysis } \\
\text { method }\end{array}$ & $\begin{array}{l}\text { Evaluation } \\
\text { method }\end{array}$ & $\begin{array}{l}\text { Metastasis } \\
\text { (yes/no) }\end{array}$ & $\begin{array}{l}\text { Tumor number } \\
\text { (single/multiple) }\end{array}$ & $\begin{array}{l}\text { Differentiation } \\
(\mathrm{W} / \mathrm{M}+\mathrm{P})\end{array}$ \\
\hline Zhou et al ${ }^{18}$ & Yes & $51 / 69$ & $\mathrm{IHC}$ & CS & $38 / 82$ & $87 / 33$ & $41 / 79$ \\
\hline Yu et $\mathrm{al}^{26}$ & Yes & $52 / 80$ & $\mathrm{IHC}$ & CS & $68 / 64$ & $84 / 48$ & $29 / 103$ \\
\hline Zhang $^{19}$ & Yes & $10 / 30$ & RT-PCR & CS & $27 / 13$ & NA & $9 / 31$ \\
\hline Ahn et $\mathrm{al}^{30}$ & Yes & $190 / 98$ & $\mathrm{IHC}$ & CS & NA & NA & NA \\
\hline Zhang et $\mathrm{al}^{27}$ & Yes & $53 / 57$ & $\mathrm{IHC}$ & CS & NA & $57 / 53$ & $24 / 86$ \\
\hline Zhang et al ${ }^{28}$ & No & $45 / 33$ & $\mathrm{IHC}$ & CS & $28 / 50$ & NA & $45 / 33$ \\
\hline Pan et $\mathrm{al}^{29}$ & No & $31 / 11$ & $\mathrm{IHC}$ & $\begin{array}{l}\text { Percentage of } \\
\text { positive cells }\end{array}$ & NA & NA & $4 / 38$ \\
\hline Studies & $\begin{array}{l}\text { Follow-up } \\
\text { (months) }\end{array}$ & $\begin{array}{l}\text { Outcome } \\
\text { indexes }\end{array}$ & $\begin{array}{l}\text { Hazard ratio } \\
(95 \% \mathrm{Cl})\end{array}$ & Notch-I (H/L) & $\begin{array}{l}\text { “High” Notch-I } \\
\text { cutoff level }\end{array}$ & Notch-I staining & $\begin{array}{l}\text { Study quality\# } \\
\text { (points) }\end{array}$ \\
\hline Zhou et al ${ }^{18}$ & 60 & OS & $2.09(1.26-3.45)$ & $64 / 56$ & $\geq 5$ & Cytoplasm membrane & $6 / 9$ \\
\hline Yu et $\mathrm{al}^{26}$ & 36 & OS & $1.39(1.07-1.81)$ & $72 / 60$ & $\geq 5$ & Cytoplasm membrane & $6 / 9$ \\
\hline Zhang ${ }^{19}$ & 31 & OS & $1.28(0.42-4.68)^{*}$ & $20 / 20$ & NA & NA & $5 / 9$ \\
\hline Ahn et $\mathrm{al}^{30}$ & 97.1 & DFS & $1.40(1.03-1.89)$ & $|45 /| 43$ & $\geq 4$ & Cytoplasm membrane & $7 / 9$ \\
\hline Zhang et al ${ }^{27}$ & NA & OS & $1.88(1.09-3.26)$ & $48 / 62$ & $\geq 5$ & Cytoplasm membrane & $5 / 9$ \\
\hline Zhang et al ${ }^{28}$ & NA & NA & NA & $52 / 26$ & $\geq 3$ & Cytoplasm & $5 / 9$ \\
\hline Pan et $\mathrm{al}^{29}$ & NA & NA & NA & $24 / 18$ & $>10 \%$ & $\begin{array}{l}\text { Cytoplasm membrane } \\
\text { nucleus }\end{array}$ & $5 / 9$ \\
\hline
\end{tabular}

Notes: *Estimated by survival curves. "Study quality was judged based on the Newcastle-Ottawa Scale (range I-9).

Abbreviations: HCC, hepatocellular carcinoma; NA, not available; W, well differentiation; $M+P$, moderate and poor differentiation; IHC, immunohistochemistry; CS, complex scoring; RT-PCR, real-time polymerase chain reaction; H, high expression; L, low expression; OS, overall survival; DFS, disease-free survival.

\begin{tabular}{|c|c|c|c|}
\hline Study ID & Meta-analysis result of OS & $\mathrm{HR}(95 \% \mathrm{Cl})$ & $\%$ weight \\
\hline Zhou et al ${ }^{18}$ & $\rightarrow$ & $2.09(1.26-3.45)$ & 9.06 \\
\hline Yu et $a^{26}$ & & $1.39(1.07-1.81)$ & 79.32 \\
\hline Zhang $^{19}$ & & $1.28(0.42-4.68)$ & 2.39 \\
\hline Zhang et $\mathrm{al}^{27}$ & - & $1.88(1.09-3.26)$ & 9.22 \\
\hline Overall $\left(I^{2}=0.0 \%, P=0.579\right)$ & & $1.50(1.17-1.83)$ & 100 \\
\hline
\end{tabular}

Figure 2 Forest plot of HR for OS of patients with HCC.

Notes: The squares and horizontal lines represent $\mathrm{HR}$ and $95 \% \mathrm{Cl}$. The diamonds represent the pooled $\mathrm{HR}$ and $95 \% \mathrm{Cl}$. The solid vertical line is at the null value. Abbreviations: HR, hazard ratio; OS, overall survival; $\mathrm{HCC}$, hepatocellular carcinoma; $\mathrm{Cl}$, confidence interval. 


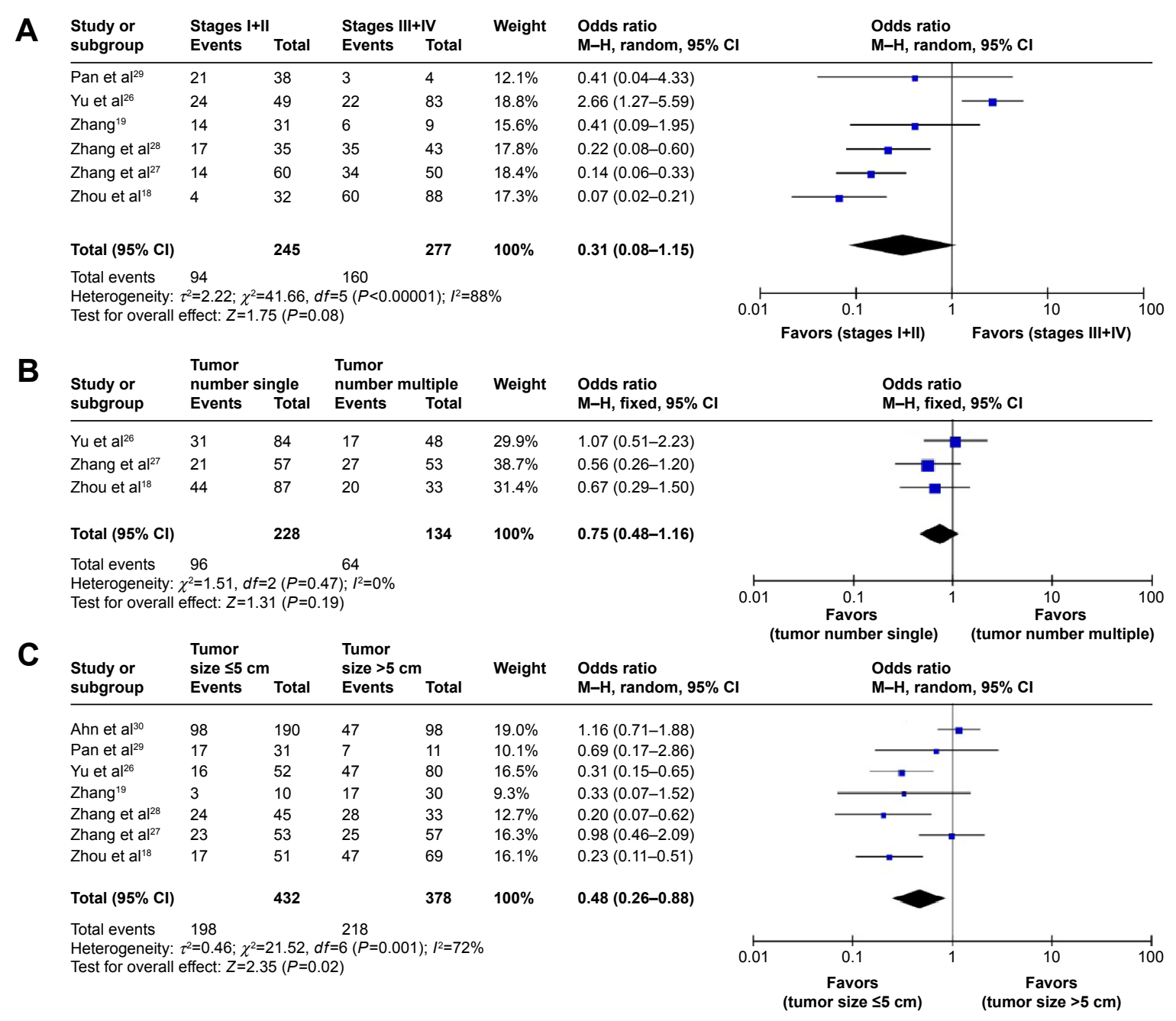

Figure 3 Association of Notch-I expression with clinicopathological parameters.

Notes: (A) The relationship between high Notch-I expression and tumor TNM stage. High Notch-I expression was not associated with TNM stages. (B) The association between high Notch-I expression and tumor number. High Notch-I expression was not associated with tumor number. (C) High Notch-I expression tended to be associated with tumor size of $>5 \mathrm{~cm}$.

Abbreviations: TNM, Tumor, Nodes, and Metastases; $\mathrm{Cl}$, confidence interval; $\mathrm{M}-\mathrm{H}$, Mantel-Haenszel.

0.16-0.86, $P=0.02$ using REM; Figure 4A). Four studies assessed the association of Notch-1 expression and portal vein invasion. ${ }^{18,26,27,30}$ The combined OR revealed no significant association between high Notch-1 expression and the presence of portal vein invasion (OR 0.46, 95\% CI 0.19-1.11, $P=0.08$; Figure 4B). Remarkable heterogeneity was found among these studies. Five studies investigated Notch-1 expression and tumor differentiation, and the pooled OR revealed that high Notch-1 expression was not associated with tumor differentiation (OR 0.68, 95\% CI 0.23-2.01, $P=0.48$ using REM; Figure 4C).

\section{Sensitivity analysis}

Sensitivity analysis was conducted to gauge the stability of the result. As shown in Table 3, when individual studies were removed sequentially, the pooled HR for OS was not significantly altered, suggesting stability of our results.

\section{Publication bias}

The reliability of results was evaluated by publication bias estimation. As shown in Figure 5, the symmetrical funnel plots revealed no evidence of publication bias for pooled OS. Furthermore, we also performed Begg's and Egger's tests for more precise assessment (Egger's test, $P=0.556$; Begg's test, $P=1.000$ ), which also implied no publication bias.

\section{Discussion}

In recent years, because of the high levels of progression and metastasis of $\mathrm{HCC}$ resulting in a dismal prognosis, numerous studies have focused on the underlying molecular mechanism in HCC metastasis. Increasing evidence proves that biomarkers can be helpful in predicting prognosis and guiding surveillance in HCC. However, such useful biomarkers have not been well identified. From a clinical perspective, therefore, it is urgent to identify new additional prognostic 


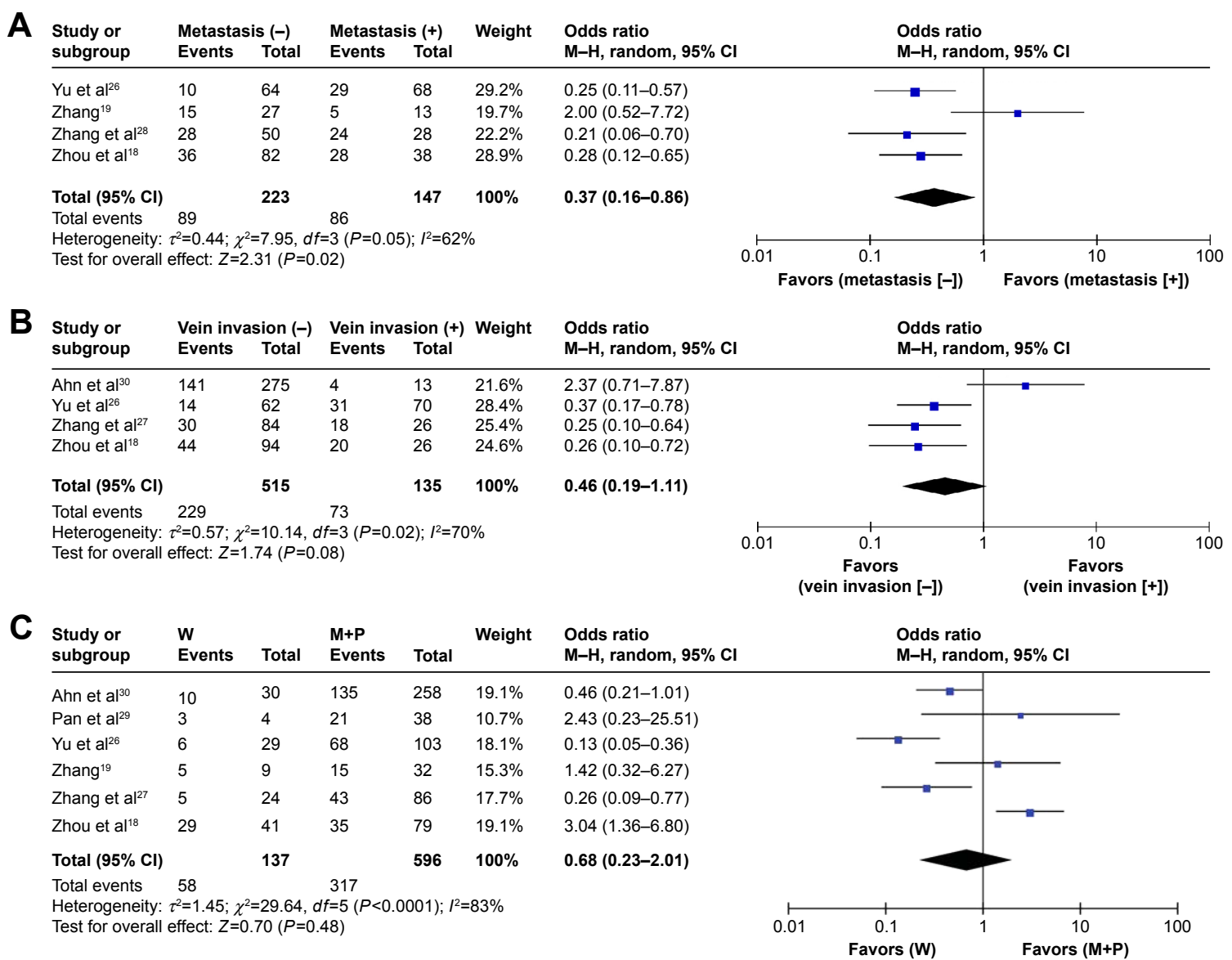

Figure 4 Association of Notch-I expression with clinicopathological parameters.

Notes: (A) High Notch-I expression was significantly associated with tumor metastasis. (B) There was no significant association between high Notch-I expression and portal vein invasion. (C) High Notch-I expression was not associated with tumor differentiation.

Abbreviations: $\mathrm{Cl}$, confidence interval; $\mathrm{M}-\mathrm{H}$, Mantel-Haenszel; $\mathrm{M}+\mathrm{P}$, moderate and poor differentiation; W, well differentiation.

and predictive markers to improve individual treatment strategies and prognosis. In this study, we introduced a potential candidate biomarker, Notch-1, for prediction of prognosis of HCC.

This study, to our knowledge, is the first meta-analysis to systematically determine the association of Notch-1 expression with clinicopathological features and prognosis of HCC. We first assessed the relationship between Notch-1 expression and OS. The pooled data indicated that high Notch-1 expression significantly predicted poor OS. Indeed, accumulating evidence indicates that the Notch signaling is involved in the initiation and progression of HCC and associated with poor clinical outcomes. ${ }^{5}$ Notch-1, as a key receptor of the

Table 3 Sensitivity analysis for OS

\begin{tabular}{lll}
\hline Outcome & Study omitted & Resulting HR $(95 \% \mathbf{C I})$ \\
\hline OS & Zhou et al $^{18}$ & $1.43(1.09-1.78)$ \\
OS & Yu et al $^{26}$ & $1.90(1.17-2.62)$ \\
OS & Zhang $^{19}$ & $1.50(1.17-1.83)$ \\
OS & Zhang et al $^{27}$ & $1.45(1.11-1.80)$ \\
\hline
\end{tabular}

Abbreviations: $\mathrm{OS}$, overall survival; $\mathrm{HR}$, hazard ratio; $\mathrm{Cl}$, confidence interval.
Notch signaling, has been reported to regulate liver cancer cell growth and invasion. ${ }^{31}$ These may partially explain the aggressive progression and dismal prognosis of HCC with high Notch-1 expression.

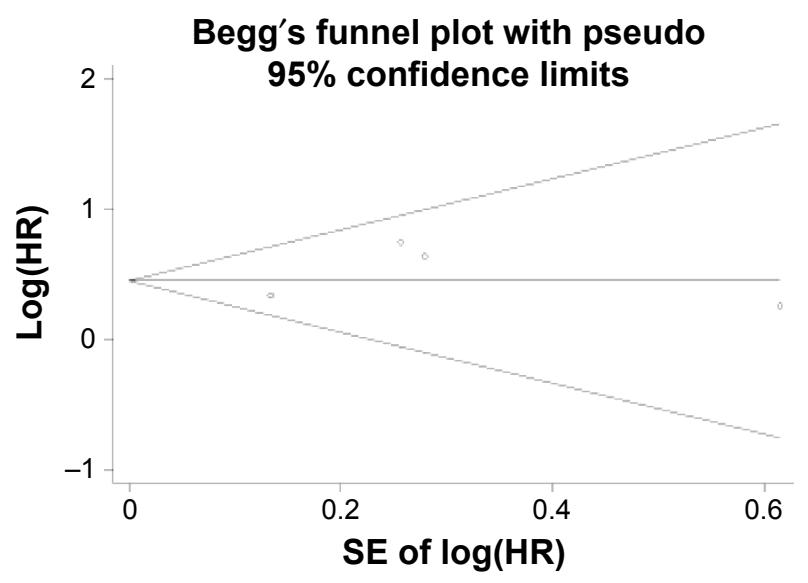

Figure 5 Publication bias analysis.

Notes: Begg's funnel plot of publication bias of HR for OS in the meta-analysis. There was no significant evidence of publication bias observed in this meta-analysis. Abbreviations: HR, hazard ratio; OS, overall survival; SE, standard error. 
In this meta-analysis, we also investigated the pooled association between Notch-1 expression and clinicopathological features. The pooled data indicated that higher Notch-1 expression was positively correlated with tumor metastasis of HCC. The results of meta-analysis supported the hypothesis that Notch-1 overexpression might contribute to malignant progression of $\mathrm{HCC}$, which subsequently leads to a poorer prognosis.

Although we conducted a systematical and comprehensive analysis, certain limitations exist and some results need to be cautiously interpreted. We estimated the HR for OS from Kaplan-Meier curves in one original study. ${ }^{19}$ These data might be less reliable than direct analysis from the original paper. The variations of the baseline characteristics of patients might have caused inherent heterogeneity within studies, affecting the interpretation of results. The data sets and total sample size are relatively limited, which might have impacted the validity of our analysis. The difference of cutoff value for judging high Notch-1 expression and criteria used to diagnose the stage and grade of HCC may have led to between-study heterogeneity. Other factors, such as the type of primary antibody and dilution, and interobserver variation, may have led to the heterogeneity of IHC studies. Although random-effects modeling and sensitivity analyses were conducted to address this heterogeneity, these statistical methods may not be sufficient. The included studies are all not of the highest quality, so the pooled results of this meta-analysis may have been affected. We assessed the publication bias and did not find significant deviation, but it is worth considering that positive results are the ones that tend to be published; thus, the association between high Notch-1 expression and poor outcome of patients with HCC might have exaggerated our evaluation.

\section{Conclusion}

In summary, although certain limitations exist, the results of current study showed that higher Notch-1 expression was associated with poorer prognosis in terms of OS of HCC. Notch-1 could be used as a useful biomarker for prediction of tumor metastasis. However, a future larger prospective study may be needed to validate our current data.

\section{Acknowledgment}

This research was supported by grants from the National Science Foundation of China (number 81402422).

\section{Disclosure}

The authors report no conflicts of interest in this work.

\section{References}

1. Ferlay J, Soerjomataram I, Dikshit R, et al. Cancer incidence and mortality worldwide: sources, methods and major patterns in GLOBOCAN 2012. Int J Cancer. 2015;136(5):E359-E386.

2. Torre LA, Bray F, Siegel RL, Ferlay J, Lortet-Tieulent J, Jemal A. Global cancer statistics, 2012. CA Cancer J Clin. 2015;65(2):87-108.

3. Meyer T. Primary liver cancer. Br J Cancer. 2013;108(4):995-996.

4. DeSantis CE, Lin CC, Mariotto AB, et al. Cancer treatment and survivorship statistics, 2014. CA Cancer J Clin. 2014;64(4):252-271.

5. Wu G, Wilson G, George J, Qiao L. Modulation of Notch signaling as a therapeutic approach for liver cancer. Curr Gene Ther. 2015;15(2): 171-181.

6. Ranganathan P, Weaver KL, Capobianco AJ. Notch signalling in solid tumours: a little bit of everything but not all the time. Nature Rev Cancer. 2011;11(5):338-351.

7. South AP, Cho RJ, Aster JC. The double-edged sword of Notch signaling in cancer. Semin Cell Dev Biol. 2012;23(4):458-464.

8. Lobry C, Oh P, Aifantis I. Oncogenic and tumor suppressor functions of Notch in cancer: it's NOTCH what you think. J Exp Med. 2011; 208(10):1931-1935.

9. Hori K, Sen A, Artavanis-Tsakonas S. Notch signaling at a glance. J Cell Sci. 2013;126(Pt 10):2135-2140.

10. Yao K, Rizzo P, Rajan P, et al. Notch-1 and notch-4 receptors as prognostic markers in breast cancer. Int J Surg Pathol. 2011;19(5):607-613.

11. Parr C, Watkins G, Jiang WG. The possible correlation of Notch-1 and Notch-2 with clinical outcome and tumour clinicopathological parameters in human breast cancer. Int J Mol Med. 2004;14(5):779-786.

12. Wang X, Song N, Zhang Y, et al. Coexpression of c-Met and Notch-1 correlates with poor prognosis in resected non-small-cell lung cancer. Tumour Biol. Epub 2015 Apr 14.

13. Donnem T, Andersen S, Al-Shibli K, Al-Saad S, Busund LT, Bremnes RM. Prognostic impact of Notch ligands and receptors in nonsmall cell lung cancer: coexpression of Notch-1 and vascular endothelial growth factor-A predicts poor survival. Cancer. 2010;116(24):5676-5685.

14. Ogawa R, Ishiguro H, Kimura M, et al. NOTCH1 expression predicts patient prognosis in esophageal squamous cell cancer. Eur Surg Res. 2013; 51(3-4):101-107.

15. Xiong Y, Zhang YY, Wu YY, et al. Correlation of over-expressions of miR-21 and Notch-1 in human colorectal cancer with clinical stages. Life Sci. 2014;106(1-2):19-24.

16. Jin HY, Zhang HY, Wang X, Xu J, Ding Y. Expression and clinical significance of Notch signaling genes in colorectal cancer. Tumour Biol. 2012;33(3):817-824.

17. Alniaimi AN, Demorest-Hayes K, Alexander VM, Seo S, Yang D, Rose S. Increased Notch1 expression is associated with poor overall survival in patients with ovarian cancer. Int J Gynecol Cancer. 2015;25(2): 208-213.

18. Zhou L, Zhang N, Li QJ, et al. Associations between high levels of Notch1 expression and high invasion and poor overall survival in hepatocellular carcinoma. Tumour Biol. 2013;34(1):543-553.

19. Zhang CD. Detection of Expression and Significance of Notch Signaling in Human Hepatocellular Carcinoma by Using the mRNA Quantitative Analysis [master's thesis]. Shijiazhuang: Heibei Medical University; 2011.

20. Liberati A, Altman DG, Tetzlaff J, et al. The PRISMA statement for reporting systematic reviews and meta-analyses of studies that evaluate health care interventions: explanation and elaboration. PLoS Med. 2009;6(7):e1000100.

21. Tierney JF, Stewart LA, Ghersi D, Burdett S, Sydes MR. Practical methods for incorporating summary time-to-event data into meta-analysis. Trials. 2007;8:16

22. Stang A. Critical evaluation of the Newcastle-Ottawa scale for the assessment of the quality of nonrandomized studies in meta-analyses. Eur J Epidemiol. 2010;25(9):603-605.

23. Higgins JP, Thompson SG, Deeks JJ, Altman DG. Measuring inconsistency in meta-analyses. BMJ (Clin Res Ed). 2003;327(7414): $557-560$. 
24. Egger M, Davey Smith G, Schneider M, Minder C. Bias in metaanalysis detected by a simple, graphical test. BMJ (Clin Res Ed). 1997; 315(7109):629-634.

25. Begg CB, Mazumdar M. Operating characteristics of a rank correlation test for publication bias. Biometrics. 1994;50(4):1088-1101.

26. Yu Y, Li X, Zhou L, et al. Clinical significance of expression of microRNA-34a and Notch1 in hepatocellular carcinoma. World Chin J Digestol. 2014;22(14):1943-1952.

27. Zhang Y, Wang DS, Zhou L, et al. Expression and significance of Notch1 in hepatocellular carcinoma. Chin J Dig Surg. 2013;12(5):378-382.

28. Zhang JG, Tian W, Yao C, Shi GS, Chen K, Zhang H. Expressions and significance of Notch1 and ADAM17 in hepatocellular carcinoma. Jiangsu Med J. 2012;38(7):820-823.
29. Pan ZK, Liang YB, Ran WW, Liu ZM, Liang J. Expressions of Notch1 and Notch3 in hepatocellular carcinoma and their clinical significance. Chin Clin Oncol. 2014;19(1):42-45.

30. Ahn S, Hyeon J, Park CK. Notch1 and Notch4 are markers for poor prognosis of hepatocellular carcinoma. Hepatobiliary Pancreat Dis Int. 2013;12(3):286-294.

31. Hu YJ, Li HY, Qiu KJ, et al. Downregulation of Notch1 inhibits the invasion of human hepatocellular carcinoma HepG2 and MHCC97H cells through the regulation of PTEN and FAK. Int J Mol Med. 2014;34(4): 1081-1086. 


\section{Supplementary material}

Table SI Newcastle-Ottawa quality assessment scale

Selection

I) Representativeness of the exposed cohort

a) Truly representative of the average $\mathrm{HCC}$ patients in the community

b) Somewhat representative of the average HCC patients in the community

c) Selected group of users (eg, nurses, volunteers)

d) No description of the derivation of the cohort

2) Selection of the nonexposed cohort

a) Drawn from the same community as the exposed cohort

b) Drawn from a different source

c) No description of the derivation of the nonexposed cohort

3) Ascertainment of exposure (proof of HCC and Notch-I measurement)

a) Secure record (eg, surgical records)

b) Structured interview

c) Written self-report

d) No description

4) Demonstration that outcome of interest was not present at start of study

a) Yes

b) No

\section{Comparability}

I) Comparability of cohorts on the basis of the design or analysis

a) Study controls for recurrence or metastasis

b) Study controls for any additional factor (age, sex, grade, tumor number, etc)

\section{Outcome}

I) Assessment of outcome

a) Independent blind assessment

b) Record linkage

c) Self-report

d) No description

2) Was follow-up long enough for outcomes to occur

a) Yes (3 years)

b) No

3) Adequacy of follow-up of cohorts

a) Complete follow-up - all subjects accounted for

b) Subjects lost to follow-up unlikely to introduce bias - small number lost $->25 \%$ follow-up, or description provided of those lost

c) Follow-up rate $<75 \%$ and no description of those lost

d) No statement

Notes: A study can be awarded a maximum of one star for each numbered item within the Selection and Outcome categories. A maximum of two stars can be given for Comparability.

Abbreviation: HCC, hepatocellular carcinoma.

\section{Publish your work in this journal}

OncoTargets and Therapy is an international, peer-reviewed, open access journal focusing on the pathological basis of all cancers, potential targets for therapy and treatment protocols employed to improve the management of cancer patients. The journal also focuses on the impact of management programs and new therapeutic agents and protocols on

\section{Dovepress}

patient perspectives such as quality of life, adherence and satisfaction The manuscript management system is completely online and includes a very quick and fair peer-review system, which is all easy to use. Visit http://www.dovepress.com/testimonials.php to read real quotes from published authors. 